\title{
LESSONS LEARNED FROM THE HUBBLE SPACE TELESCOPE (HST) CONTAMINATION CONTROL PROGRAM
}

\author{
Patricia A. Hansen ${ }^{1}$ \\ Jet Propulsion Laboratory, California Institute of Technology \\ Jacqueline A. Townsend and Randy J. Hedgeland \\ NASA/Goddard Space Flight Center
}

\begin{abstract}
Over the past two decades, the Hubble Space Telescope (HST) Contamination Control Program has evolved from a ground-based integration program to a space-based science-sustaining program. The contamination controls from the new-generation Scientific Instruments and Orbital Replacement Units were incorporated into the HST Contamination Control Program to maintain scientific capability over the life of the telescope. Long-term onorbit scientific data has shown that these contamination controls implemented for the instruments, Servicing Mission activities (Orbiter, Astronauts, and mission), and on-orbit operations successfully protected the HST from contamination and the instruments from self-contamination.
\end{abstract}

\section{INTRODUCTION}

The Hubble Space Telescope (HST) Contamination Control Program evolved over the past 15 years going from a ground-based integration program to a space-based science-sustaining program. Since the HST was deployed in April 1990, it has been serviced four times, approximately every three years. During these servicing missions, the telescope's science capability was upgraded with the installation of second- and third-generation scientific instruments. For each servicing mission, the contamination control program was assessed for its applicability to the mission complement and adjustments were made depending on the sensitivity of the scientific instruments and other orbital replacement units and the demands of the servicing tasks.

Due to the extreme contamination sensitivity of the HST, scientific instruments, and Fine Guidance Sensors to molecular and particulate contamination, all aspects of a servicing mission were assessed for potential and subsequent contamination effects to the specific contamination-sensitive surface (optical and thermal). The assessment began with the basic requirements for the telescope and extended to each mission component. Multimission hardware, including the servicing mission carriers, Scientific Instrument Protective Enclosures and Orbital Replacement Unit Protective Enclosures, were unique as they themselves are not contamination sensitive, but provide a "safe haven" for hardware that was extremely sensitive to molecular and particulate contamination. This multi-mission hardware essentially isolated the contamination-sensitive hardware from the Orbiter payload bay and its contamination environment. However, because of the large surface area of the servicing carriers, both outgassing levels and surface cleanliness levels were controlled during all aspects of integration, test, launch activities, and onorbit operations.

The HST Contamination Control Program, while specific to HST and the Orbiter, offers many lessons learned that are applicable to other contamination-sensitive hardware. When effectively applied across contamination-sensitive spacecraft, these lessons may reduce the cost of a contamination control program from the standard 10 percent of the program costs to $1-2$ percent of the program cost (1). This paper gives a brief overview of the HST servicing mission hardware, the Servicing Mission Contamination Control Program, and the lessons learned from this long-term, highly successful program. The details for each of the mission-unique HST Servicing Mission Contamination Control Programs can be found in References 2-9.

\footnotetext{
${ }^{1}$ Formerly of the NASA/Goddard Space Flight Center
} 


\section{HUBBLE SPACE TELESCOPE}

The HST was designed to be periodicaily serviced on-orbit during its 20-year mission. The HST science observations are accomplished through five science instruments (four axial and one radial) and three Fine Guidance Sensors (FGS). To-date the HST has had four successful servicing missions occurring approximately every 2-3 years (see Table 1) during which critical systems and science capabilities have been upgraded during extra-vehicular activities (EVAs) or "space walks". These activities presented an inherent risk to the cleanliness of the telescope and will be discussed later in this paper. However, a comprehensive contamination control program addressing the Orbiter, the Astronauts, and the carrier hardware, was developed and implemented for each of the servicing missions. This effort was extremely successful and, to-date, no on-orbit contamination events have been reported.

Table 1. HST Servicing Missions

$\begin{array}{ccc}\text { Mission } & \text { Designation } & \underline{\text { Year }} \\ \text { Deploy } & \text { STS-31 } & \text { April 1990 } \\ \text { SM1 } & \text { STS-61 } & \text { December 1993 } \\ \text { SM2 } & \text { STS-82 } & \text { February 1997 } \\ \text { SM3A } & \text { STS-103 } & \text { December 1999 } \\ \text { SM3B } & \text { STS-109 } & \text { March 2002 }\end{array}$

\section{ON-ORBIT SERVICING}

The HST was designed to be periodically serviced on-orbit using the Space Transportation System (STS). Servicing mission carriers provided an interface from the Orbiter to the scientific instruments, orbital replacement units, and the captured telescope. The servicing carriers were configured for each mission to accommodate missionunique orbital replacement units; the basic carrier (structure and support airborne flight equipment) remained unchanged. The HST servicing carriers which were flown during each of the servicing missions are listed in Table 2.

\section{Servicing Carriers}

The HST servicing carriers included: the Solar Array Carrier (SAC), the Orbital Replacement Unit Carrier (ORUC), the Flight Support System (FSS), the Rigid Array Carrier (RAC), the Second Axial Carrier (SAC), and the Multi-Use Lightweight Experiment (MULE). The servicing mission carrier manifest is illustrated in Table 2. As can be seen the Flight Support System and the Orbital Replacement Unit Carrier were manifested most often, requiring the most number of configuration changes to support specific hardware manifested for a specific servicing mission. For the Orbital Replacement Unit Carrier, this primarily involved the reconfiguration of its shelves and the Orbital Replacement Unit Protective Enclosures. The other carriers - Multi-Use Lightweight Explorer, Rigid Array Carrier, Second Axial Carrier, and Solar Array Carrier - were manifested for specific hardware elements and as such were flown less often. 
Table 2. Carrier Mission Manifest

\begin{tabular}{|c|c|c|c|c|}
\hline Carrier & SM1 & SM2 & SM3A & SM3B \\
\hline Flight Support System & $\checkmark$ & $\checkmark$ & $\checkmark$ & $\checkmark$ \\
\hline Multi-Use Lightweight Explorer & & & & $\checkmark$ \\
\hline Orbital Replacement Unit Carrier & $\checkmark$ & $\checkmark$ & $\checkmark$ & \\
\hline Rigid Array Carrier & & & & $\checkmark$ \\
\hline Second Axial Carrier & & $\checkmark$ & & $\checkmark$ \\
\hline Solar Array Carrier & $\checkmark$ & & & \\
\hline
\end{tabular}

The $15^{\prime}$ long x 15' wide x 15' high Solar Array Carrier (Figure 1) functioned as the load isolation system for the Solar Array 2 during the First Servicing Mission. For the Second Servicing Mission, the Solar Array Carrier was reconfigured, renamed the Second Axial Carrier, and provided a load isolation system for the Axial Scientific Instrument Protective Enclosure which in turn provided a contamination and thermally-controlled environment for the Near Infrared Cosmic Origins Spectrograph (NICMOS).

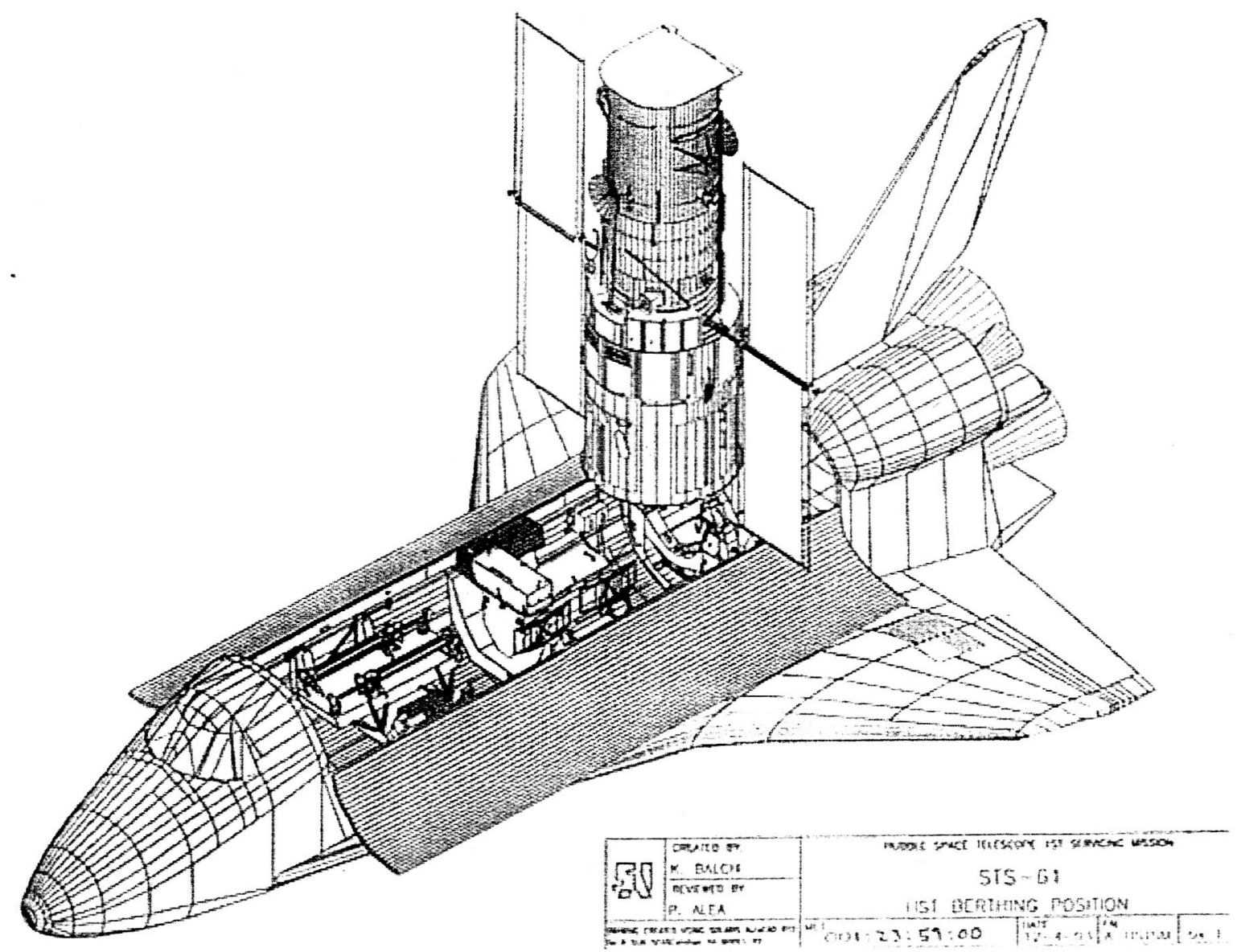

Figure 1. Servicing Mission 1 Hardware Configuration. The servicing carrier hardware is shown manifested in the Orbiter payload bay. The Solar Array Carrier is shown in the foreground, followed by the Orbital Replacement Unit Carrier. The HST is shown berthed on the Flight Support System. 
The most contamination sensitive carrier is the $12^{\prime}$ long $\mathrm{x} 15^{\prime}$ ' wide $\mathrm{x} 15^{\prime}$ high Orbital Replacement Unit Carrier (Figures 1 and 2). The Orbital Replacement Unit Carrier provided a load isolation system for an Axial Scientific Instrument Protective Enclosure (SIPE) and the Radial SIPE. These SIPEs, collectively known as the BISIPE, provided a contamination- and thermally-controlled environment for stowed scientific instruments. For the First Servicing Mission, the Corrective Optics and Space Telescope Axial Replacement (COSTAR) and Wide Field and Planetary Camera II (WFPC-II) were stowed in the Axial and Radial SIPEs, respectively. For the Second Servicing Mission, the Axial SIPE held the Space Telescope Imaging Spectrograph (STIS) and the Radial SIPE was reconfigured to hold a Fine Guidance Sensor and was renamed the Fine Guidance Sensor SIPE. Because of the optical sensitivity of the scientific instruments and Fine Guidance Sensors, the Second Axial Carrier and the Orbital Replacement Unit Carrier were the most contamination-sensitive carriers.

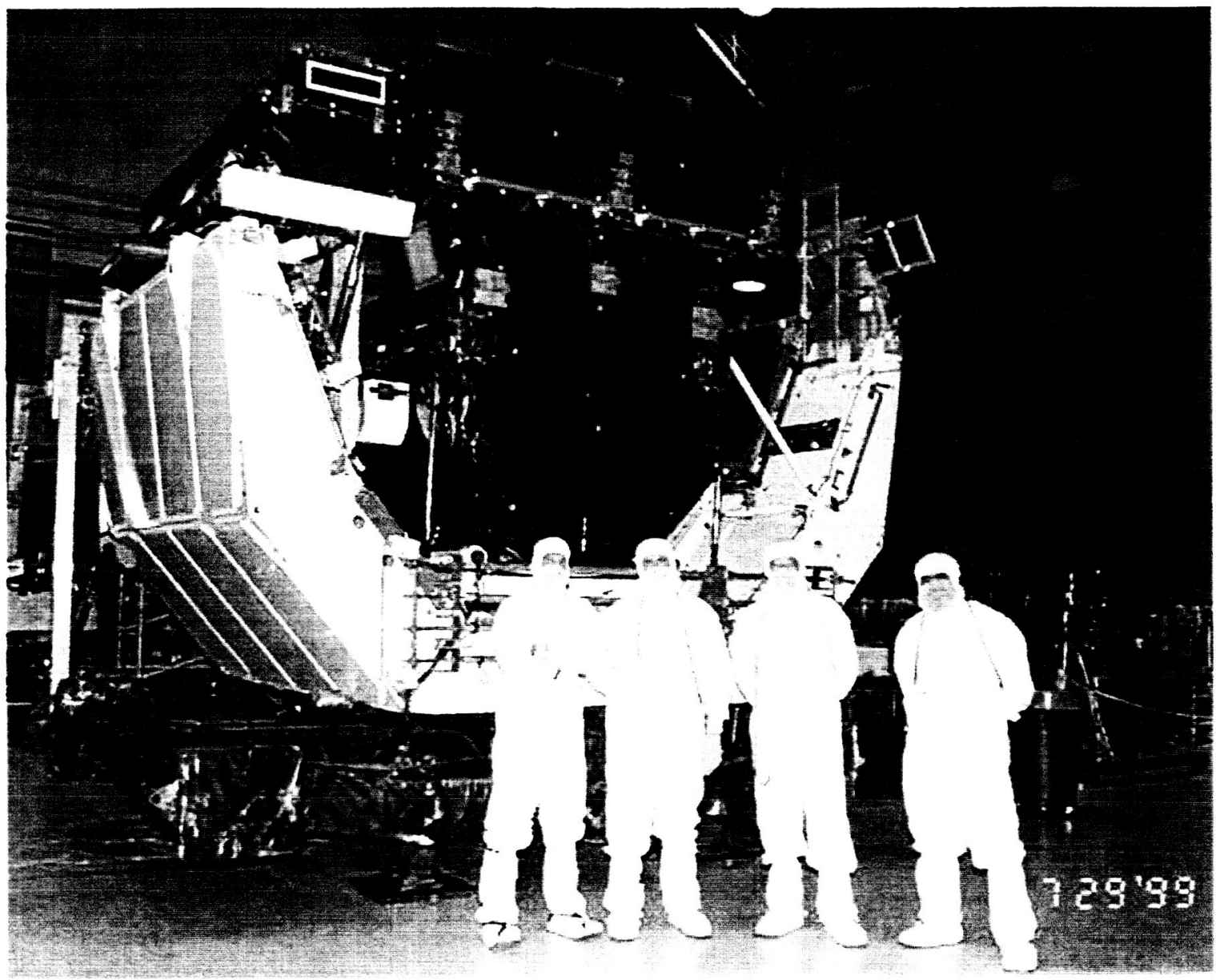

Figure 2. The Orbital Replacement Unit Carrier. The Orbital Replacement Unit Carrier is shown with the Fine Guidance Sensor SIPE forward. Orbital Replacement Unit Protective Enclosures are shown on the shelf (left).

The SIPEs provided a thermal environment equivalent to that inside the HST. The warm thermal environment ensured that the scientific instruments and Fine Guidance Sensors remained within their temperature limits during the EVA. This also ensured that any outgassing inside the SIPEs, which would otherwise have affected the optical performance, would not condense on the scientific instruments or Fine Guidance Sensors. The SIPEs also provided a purge interface, which allowed the scientific instruments and Fine Guidance Sensors to be purged until launch $(\mathrm{T}-0)$. Vent restrictor plates $(37 \mu \mathrm{m}$ mesh) inhibited particulate contamination of the scientific instruments or Fine Guidance Sensors during all ground and launch activities. Additional details about the SIPE design, test, and qualification for flight can be found in References 5-6. 
The 5' long x 15' wide $x 15$ ' high Flight Support System was used as the maintenance platform to berth the HST to the Orbiter during the EVAs (see Figure 1). The 5' long x 15' wide $\mathrm{x}$ 15' high Multi-Use Lightweight Explorer provided stowage for orbital replacement units (see Figure 3). The 12' long x 15' wide x 15' high Rigid Array Carrier was used during Servicing Mission 3B to stow the Rigid Solar Arrays (see Figure 3). Once the Rigid Solar Arrays were integrated onto the HST during the servicing mission, the Rigid Array Carrier was used to stow the de-integrated Solar Array-2 during the rest of the mission (including descent).

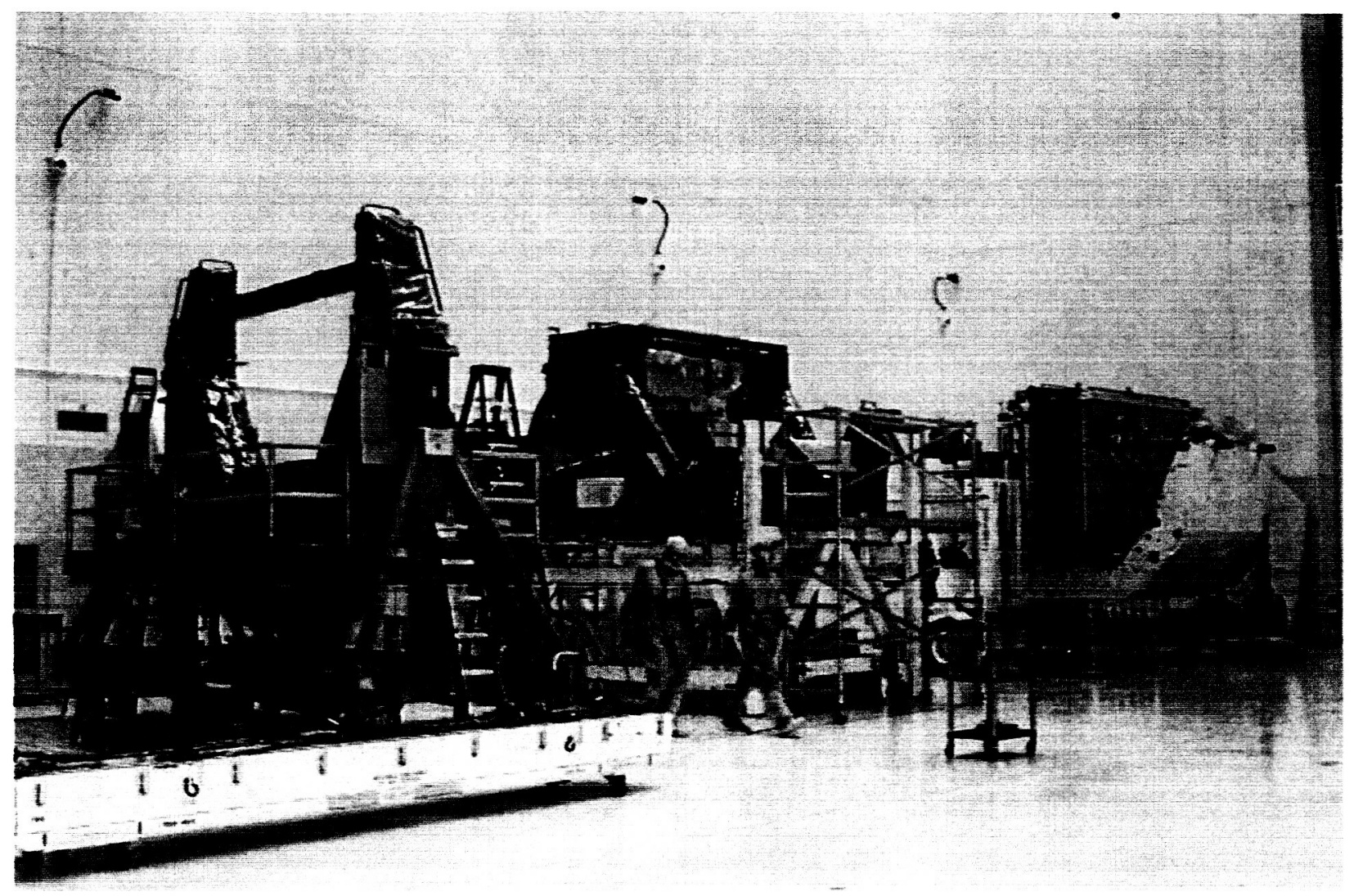

Figure 3. The Servicing Mission 3B servicing carriers -Multi-Use Lightweight Explorer (left foreground), Second Axial Carrier (center), and Rigid Array Carrier (right). The Axial Scientific Instrument Protective Enclosure (large rectangular box) is shown mounted on the Second Axial Carrier. The Rigid Solar Arrays are shown stowed on the Rigid Array Carrier.

Due to the diversity of the orbital replacement units and scientific instruments manifested for each flight, the carriers provided the most flexible stowage capability for the servicing mission hardware. Because of the planned multiple mission use of the Orbital Replacement Unit Carrier and the Flight Support System over a decade, the HST contamination control program looked at the "big picture" to determine the most cost effective contamination control approach that provided the needed contamination controlled environment for the scientific instruments and Fine Guidance Sensors while controlling cost. Because of the excessive cost and schedule required to recertify the molecular outgassing levels of the individual carriers for each servicing mission, the HST contamination control program looked at innovative methods to alleviate the recertification of the carriers for each mission. Controlling the material added to the carriers and individually certifying new hardware prior to integration onto the carrier accomplished this. The storage, integration and test environment was also controlled, with the carriers spending the majority of these activities in a Class 10,000 (M5.5 or ISO Class 7) cleanroom. When not in a cleanroom, the carriers were double bagged. During storage, the carriers were cleaned periodically to maintain the surface cleanliness levels. 


\section{SERVICING MISSION CONTAMINATION PROGRAM}

The servicing missions were complex and required that the telescope be exposed to the Orbiter (including servicing carriers) environment during the installation of the scientific instruments and Fine Guidance Sensors into the HST Aft Shroud. This exposure was typically from one to seven hours during an EVA. Over the duration of all the servicing missions the telescope was exposed for approximately 60 hours. During the scientific instrument installation, one EVA crew member (i.e., an astronaut) entered the Aft Shroud to guide both the old instrument out of the telescope and the new instrument into the telescope. Because of this exposure and to maintain the Ultraviolet (UV) capabilities of the telescope, the contamination requirements placed on both the Orbiter and carriers were quite stringent. While one might argue that the scientific instrument was the most contamination sensitive element, in reality, maintaining the low contamination flux in the telescope's optical path was the servicing mission's primary contamination requirement.

Neither the Orbiter nor the Extravehicular Mobility Unit (space suit) contamination levels was verified by methods other than visual examination. Outgassing levels were not measured and by the nature of the Orbiter, many materials generally not used around sensitive hardware were used for performance. Where possible, materials which were verified to be high outgassing, which would not impact the Orbiter performance were removed for the HST servicing missions. In addition, a best effort was made to control contamination during Orbiter processing activities.

Ground processing activities, Orbiter integration and the overall mission activities were assessed for subsequent contamination to the HST and the scientific instruments and Fine Guidance Sensors for each servicing mission. The assessment began with the basic requirements for the HST and extended to each mission component. An overall contamination budget was established allocating acceptable degradation among mission phases. The servicing mission cleanliness requirements and budgets were set with respect to hardware line-of-sight views of sensitive surfaces, purging of the scientific instruments for sustaining critical element functional lifetimes, Orbiter and EVA effects, Orbiter cleanliness, cleanroom protocol, and launch site (Kennedy Space Center) integration activities.

Prior to each servicing mission, the HST contamination control philosophy was reviewed to determine its applicability to reflown carrier hardware, new scientific instruments, new orbital replacement units, and HST optical performance. The current contamination control program evolved over four servicing missions. The new carriers, scientific instruments, Fine Guidance Sensors, orbital replacement units, and significantly reworked contaminationsensitive hardware, such as the SIPEs, were certified to the required outgassing rate prior to a servicing mission.

\section{Telescope and Scientific Instrument Contamination Requirements}

To maintain the UV performance of the telescope and, therefore, the scientific instruments, the telescope contamination requirements addressed both the surface level cleanliness of the Primary and Secondary mirrors and the allowable outgassing flux rate for the telescope's optical path (known as the hub area). The scientific instrument requirements were based on the optical sensitivity of the scientific instrument to contamination.

\section{Primary and Secondary Mirrors}

The particulate contamination requirements were less than five percent maximum area coverage for the summation of the Primary and Secondary Mirrors at end-of-life (EOL). This was determined pre-launch by measuring the obscuration ratio of optical witness samples. To date, no scientific instrument data has indicated that this requirement has been violated.

The molecular contamination requirement was less than a 10 percent decrease in reflectance at LymanAlpha (1216 Angstrom) wavelengths on the Primary and Secondary Mirrors after five years on-orbit. This was determined pre-launch by measuring optical witness mirrors. Neither integrated nor periodic measurements indicated that this requirement had been violated prior to the telescope's deployment. The initial outgassing criteria was $4.33 \times 10^{-13} \mathrm{~g} / \mathrm{cm}^{2}$-s flux as measured with the mirrors at nominal operating temperatures and the collector at 
$-20^{\circ} \mathrm{C}$. The optical witness mirror reflectance degradation was also required to be less than three percent at LymanAlpha wavelengths $(1216 \AA)$.

Hub Area

The light path of the telescope is referred to as the "hub area". The four axial and one radial scientific instrument apertures, the three Fine Guidance Sensor apertures and the back of the Primary Mirror define this area. To control the amount of contamination entering this area and to prevent cross contamination, contamination requirements were flowed down to the scientific instruments and Fine Guidance Sensors. The outgassing rate from an instrument aperture or Fine Guidance Sensor aperture into the hub area could not exceed $1.32 \times 10^{-9} \mathrm{~g} / \mathrm{sec}$. For the upgraded Fine Guidance Sensors which were installed during the Servicing Mission 2 and Servicing Mission 3A, the Fine Guidance Sensor's outgassing rate was measured with the instrument at worse case hot operational temperatures (approximately $25^{\circ} \mathrm{C}$ ) and the collector at $-65^{\circ} \mathrm{C}$. Similarly, the surface level contamination requirements for any item entering the telescope were Level 400B per MIL-STD-1246 (currently IEST-STDCC1246D; Reference 14).

\section{Aft Shroud}

Four axial scientific instruments were installed in the Aft Shroud. To control the amount of contamination entering this area and to prevent cross contamination, the scientific instruments met minimum surface levels cleanliness and outgassing requirements. The scientific instrument exterior surface cleanliness level did not exceed Level 400B per MIL-STD-1246 (currently IEST-STD-CC1246D). The outgassing requirement measured at the scientific instrument aft vent could not exceed an equivalent rate of $1.56 \times 10^{-9} \mathrm{~g} / \mathrm{hr}-\mathrm{cm}^{2}$ based on the exterior surface area of the instrument. This outgassing rate was measured with the scientific instrument ten degrees above the worse case predicted hot operational temperatures and the collector at $-20^{\circ} \mathrm{C}$. While the largest percentage of the outgassed products from the scientific instruments was vented through the telescope's aft vents, there was a small probability that a scientific instrument could increase the contamination flux in the hub area, thus affecting the telescope's performance. These requirements also controlled cross-contamination between the scientific instruments and Rate Sensor Units (gyroscopes).

The Rate Sensor Units resided in one of the Aft Shroud Bays (between two axial scientific instruments). Although not inherently contamination sensitive (as a Rate Sensor Unit versus individual gyroscopes), the Rate Sensor Units were subjected to the same stringent contamination requirements as the scientific instruments. This included outgassing and surface cleanliness levels and minimized cross-contamination between the scientific instruments and the Rate Sensor Units.

\section{Scientific Instruments and Fine Guidance Sensors}

As shown in Table 3, five second- and third-generation scientific instruments and two upgraded Fine Guidance Sensors have been installed into the telescope during the servicing missions. The scientific instruments and Fine Guidance Sensors had individual contamination requirements based on their optical sensitivity. For example, scientific instruments viewing in the UV wavelength regions would have the most sensitivity to molecular contamination. However, scientific instruments viewing in the infrared wavelength regions would have the greatest sensitivity to particulate contamination. The instrument contamination requirements have been reported elsewhere (2-8). The scientific instruments and Fine Guidance Sensors were delivered to NASA with verification of internal contamination levels. These levels were maintained throughout the integration, test, and launch activities through contamination controls such as a gaseous Nitrogen purge. 
Table 3. Scientific Instrument/Orbital Replacement Unit Installation

Scientific Instrument/Orbital Replacement Unit

Wide Field and Planetary Camera II (WFPC-2)

Corrective Optics and Space Telescope Axial Replacement (COSTAR)

(3) Rate Sensor Unit

Space Telescope Imaging Spectrometer (STIS)

Near Infrared Camera and Multi-Object Spectrometer (NICMOS)

Fine Guidance Sensor R-1

(3) Rate Sensor Unit

Fine Guidance Sensor R-2

(3) Rate Sensor Unit

Advanced Camera for Surveys

(3) Rate Sensor Unit
Date of Installation

December 1993

December 1993

December 1993

February 1997

February 1997

February 1997

February 1997

December 1999

December 1999

March 2002

March 2002

\section{Orbiter and EVA Effects}

In addition to many hardware cleanliness requirements, numerous analyses were performed for the Orbiter environment and EVA contamination impacts. These analyses provided critical assessments for controlling on-orbit contamination generating activities and provided the necessary quantitative details for imposing ground processing requirements for the Orbiter. The major analyses included plume impingement, waste/water dumps, SIPE, Extravehicular Mobility Unit (space suit), Orbiter reboost, and HST configuration changes including deployed solar arrays. These analyses represent the core of the cleanliness concerns associated with the shuttle and EVAs.

In addition to the analyses for the Orbiter, cleaning requirements were assessed and levied on the Orbiter payload bay. To quantify the effects of the crew compartment on subsequent EVAs relative to the particulate environment, two witness plates were flown on STS-51 to characterize the particle transfer from the Extravehicular Mobility Unit during Astronaut translation through the Orbiter payload bay and during EVA activities. These results were used to determine the crew cabin and Extravehicular Mobility Unit cleanliness requirements (10).

The analyses of the Orbiter plume impingement assessed the degradation of the HST surfaces due to gaseous and liquid droplet impingement from thruster firings during maneuvers and station-keeping operations. Byproducts from the incomplete combustion, such as monomethyl hydrazine (MMH)-nitrate, could have had detrimental effects on contamination-sensitive and thermal control surfaces. The station-keeping and attitude adjustments considered were low- $Z$ and norm- $Z$ modes. Because the byproduct mass flux in the norm- $Z$ thruster firing case was significant, limitations were imposed for Orbiter operations.

Significant droplets were formed during the Orbiter waste/water exhaust. These droplets may have posed a potential threat to the HST during EVA operations when the telescope's Aft Shroud doors were open. The estimation of the maximum effluent released during these dumps was approximately $320-1 \mathrm{bm}$ for each dump. Since this represented a significant amount of released material during the HST servicing operations, restrictions were placed in the flight rules for all of the servicing missions. All dumps were constrained 120 minutes prior to and during EVAs to preclude potential impingement on critical areas of the HST.

Because the SIPEs provided cleanliness protection during launch, ascent, and on-orbit operations for the scientific instruments, a separate analysis was performed to assess contamination impacts. The primary objective was to examine impacts due to particle redistribution within the SIPEs, molecular flow, and moisture control with 
the SIPEs. All of the elements of this analysis accounted for any degradation to the scientific instruments during these phases. The results of these analyses are discussed in References 5-6.

During an EVA, the amount and type of contamination emitted by an astronaut was considered a threat to optical surfaces on the HST. In addition, the astronaut was in close proximity (e.g., line-of-sight) to the scientific instruments and Aft Shroud. The main concern was contamination contributions from the Extravehicular Mobility Unit (space suit). The Extravehicular Mobility Unit exhaust was analyzed and assessed for molecular and particulate contributions. The main byproduct of the Extravehicular Mobility Unit exhaust was estimated to be 1 to $1.5 \mathrm{lb} / \mathrm{hr}$ of water vapor and/or ice. Because the sensitive HST surface temperatures were above the water condensation temperature for a low-pressure environment, no contaminant depositions from the Extravehicular Mobility Units were expected.

\section{Orbiter Payload Bay Cleanliness Requirements}

The Orbiter payload bay liner and thermal control blankets (forward and aft bulkheads; Bays 12 and 13) provided thermal control to the payload and may be flown on many missions. A reflown liner section or thermal control blankets could have been a large outgassing source to a payload if contaminated by a previous payload or another mission. As this potential outgassing source could not be quantified (the outgassing species and rate), a new unflown payload bay liner was requested for the entire payload bay. The thermal blankets could not be replaced due to excessive cost; however, they were cleaned with an isopropyl alcohol (IPA)/deionized (DI) water mixture and verified to have no significant fluorescing molecular contamination. Small amounts of molecular contamination could have been tolerated, but were evaluated on a case-by-case basis and were dependent on their location within the payload bay.

Based on the hardware cleanliness requirements, for the servicing missions a new payload bay liner was cleaned to visibly clean highly sensitive (VCHS) per Johnson Space Center Document Number SN-C-0005C, with an IPADI water mixture. During Orbiter servicing in the Orbiter Processing Facility (OPF), the payload bay liner and thermal blankets, including the bilge area and wire trays, were vacuumed every three days. The payload bay was cleaned to VCHS at the Payload Changeout Room (PCR), with the Orbiter in the vertical configuration. Vertical cleaning at the Pad provided both the best access to all levels and a top-down cleaning approach so that any particles cleaned from the level above, but not captured, would fall to a level which would be subsequently cleaned. Again, the thermal blankets were verified to have no significant fluorescing molecular contamination.

\section{Cleanroom Protocol}

The biggest contamination threat to the servicing mission carriers, scientific instruments, and Fine Guidance Sensors was the personnel working on or around them. To control this threat, the servicing mission carriers spent the majority of their time in a Class 10,000 (M5.5 or ISO Class 7) cleanroom. The cleanroom protocol, detailed in Reference 7, was derived from the hardware requirements, contamination control practices, and data from previous missions. Personnel constraints, cleanroom operating procedures, and site management issues were addressed for each facility in which the servicing mission hardware was assembled, integrated or tested. Activities which had the potential to contaminate hardware were identified and controlled by procedure. These activities included crew familiarizations, alignment and envelopment measurements with the High Fidelity Mechanical Simulator and scientific instrument to SIPE fit-checks and integration.

Launch site integration activities also presented a challenge to maintaining the servicing carriers, scientific instrument and Fine Guidance Sensor contamination levels. Because of the servicing mission carriers' physical size, the launch site could only provide Class 100,000 (M 6.5 or ISO Class 8) facilities. However, Class 10,000 (M5.5 or ISO Class 7) cleanroom protocols were used which resulted in significantly lower operating levels - Class 10,000 to Class 20,000 during typical integration activities. During the scientific instrument insertion into the SIPE, the cleanroom was run at Class 10,000 (M5.5 or ISO Class 7) cleanroom with strict personnel limits. For the servicing missions, these cleanroom protocols resulted in hardware contamination levels significantly below required limits (7). 


\section{SCIENTIFIC INSTRUMENT DATA}

\section{Post-Mission Data}

Each of the scientific instruments returned from orbit underwent extensive post-mission inspection and testing. The most significant findings, from a contamination perspective, were from the Wide Field and Planetary Camera I (WFPC-1). These results reported elsewhere (11-12), significantly impacted the Servicing Mission Contamination Control Program and are summarized for completeness.

The WFPC-1 was one of the original scientific instruments deployed on HST. It was replaced with the Wide Field and Planetary Camera II (WFPC-2) during the First Servicing Mission, which provided upgraded science capability. Post-mission inspection of the WFPC-1 pick-off mirror (see Figure 4) showed some haziness when viewed with a bright light. A Failure Review Board was convened to determine the cause of the haziness and to recommend remedial action to the HST Project to preclude contamination of other optical surfaces.

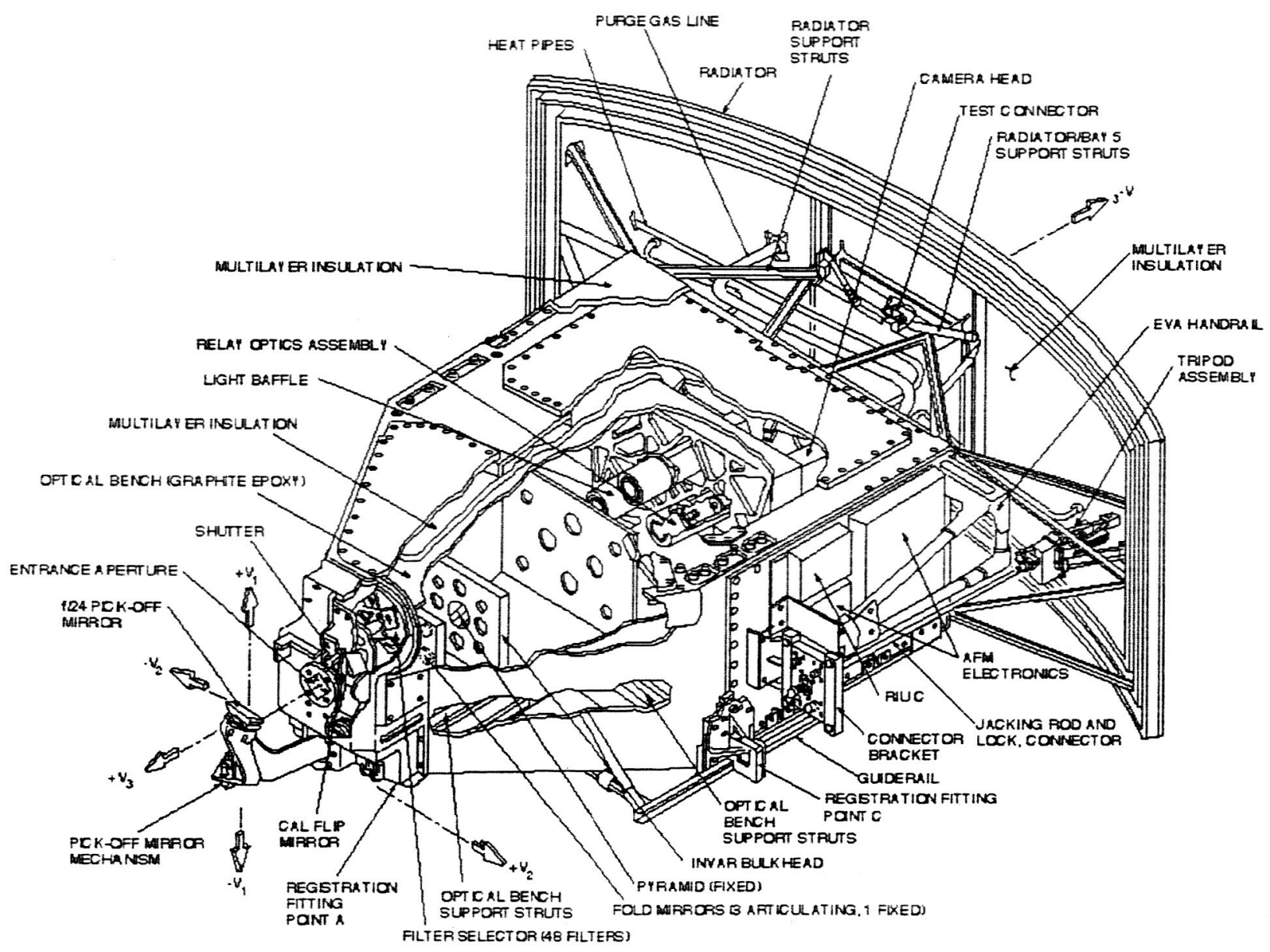

Figure 4. Wide Field and Planetary Camera II. The Wide Field Planetary Camera II is shown; however the optical train and overall instrument layout was identical to that of the Wide Field Planetary Camera I.

The WFPC-1 pick-off mirror's reflectance was severely degraded in the far UV region when compared with pre-launch measurements and measurements from the flight-spare pick-off mirror (11-12). Subsequent testing 
of the mirror indicated that there was a partially photopolymerized contaminant layer which was approximately 450 $\AA$ thick (11). It was theorized that the photopolymerization of contaminants occurred when the pick-off mirror was exposed to Earth-albedo Ultraviolet radiation. Most interesting, was the fact that this contaminant layer caused no change in reflectance at wavelengths above $2000 \AA$. See Reference 11 for a complete discussion of the WFPC- 1 post-mission analyses. It should be noted that for the scientific instruments installed during the Second Servicing Mission, WFPC-2 and Corrective Optics and Space Telescope Axial Replacement (COSTAR), there was no subsequent degradation of their optical performance in the UV wavelength region $(1216 \AA-2000 \AA)$.

The WFPC-1 (Magnesium Flouride) window, which sealed the WFPC-1 instrument, also had a partially photopolymerized contamination layer on the outside (facing the HST optical path) surface. Its thickness was approximately $150 \AA$ thick. Filters from the returned High Speed Photometer (HSP) also had a $150 \AA$ thick contaminant layer.

Forensic evidence of the HST build and contamination control program indicated that three Fine Guidance Sensors deployed with the telescope were not held to the same rigorous materials screening criteria, outgassing rate, or surface cleanliness levels as the scientific instruments. This was an oversight that was subsequently corrected for all replacement Fine Guidance Sensors. The Failure Review Board concluded that the Fine Guidance Sensors were most likely responsible for most of the contamination seen on the WFPC-1 pick-off mirror (11). However, telescope surfaces (structural members supporting the Aft Shroud and Primary Mirror) were also a contributor (11). The original scientific instruments were not considered likely sources of contamination to the WFPC-1 pick-off mirror as they all underwent extensive thermal-vacuum testing and their outgassing rates were well characterized.

\section{On-Orbit Data}

\section{Goddard High Resolution Spectrometer}

The Goddard High Resolution Spectrometer (GHRS) was one of the original HST scientific instruments and the only scientific instrument that measured at Lyman-Alpha (1216 $\AA$ ) wavelengths. Over its life (1990-1997) it did not measure a decrease in sensitivity greater than its three percent uncertainty at $1216 \AA$. However, its channel at $1150 \AA$, showed decreases in sensitivity slightly above its 10 percent uncertainty.

Corrective Optics and Space Telescope Axial Replacement (COSTAR)

The Corrective Optics and Space Telescope Axial Replacement (COSTAR) was installed during the First Servicing Mission and provided corrective optics to the three remaining axial scientific instruments. No degradation of the corrective optics performance was reported (1993-2002).

\section{Space Telescope Imaging Spectrograph}

The Space Telescope Imaging Spectrograph (STIS) was one of the HST second-generation scientific instruments. While the post-mission analyses of the WFPC-1 occurred, STIS was undergoing its final instrument integration and test phase. Because of the sensitivity of the STIS mirrors to only small amounts of contaminants (the end-of-life requirement was less than $15 \AA$ per optical element); the STIS contamination control program incorporated additional pre-processing of large amounts of non-metallic materials. Most important were the contamination controls during the on-orbit operations and the avoidance of the contamination-sensitive optics to the Earth-albedo Ultraviolet radiation and potential photopolymerization of contamination on the STIS mirrors. Due to this concern, the STIS design was changed to accommodate a shutter at its aperture which was closed during each Earth pass. The STIS contamination control program was discussed in Reference 9.

The STIS was installed into the HST during the Second Servicing Mission (February 1997). On-orbit calibration data, taken after each servicing mission (SM2, SM3A, and SM3B) indicated that the STIS CCD sensitivity at Lyman-Alpha $(1216 \AA)$ wavelengths has changed on-average approximately one percent per year. In 2002 , during the SM3B post-servicing mission calibration cycle, the wavelength-averaged sensitivity loss remained 
at approximately one percent per year (13). This data indicated that over the STIS 5-year mission, the CCD sensitivity had declined approximately five percent.

This STIS on-orbit data indicated that the contamination levels within the STIS instrument were low and most likely, were due to monolayer contamination depositions. The data also indicated that the sensitivity loss remained constant over the STIS mission (1997- to present) also indicating that the contamination levels within the HST Aft Shroud were low. The requirements that were set for the instrument were met, which allowed the STIS to continue its science operations past its 5 -year mission.

\section{LESSONS LEARNED}

The Lessons Learned are grouped together by general guidelines, multi-mission hardware, and scientific instrument. The general guidelines are universal and are applicable to all contamination-sensitive programs. The multi-mission hardware guidelines are less general, but may be applied to programs where multiple buses are built and stored prior to use. Finally, the scientific instrument guidelines are specific to contamination-sensitive hardware. However, many of these guidelines would be applicable at the spacecraft level, depending on the configuration of the spacecraft and contamination-sensitive instrument(s).

\section{General}

1. Develop an overall contamination control program. Flow down the requirements into contamination control plans, handling procedures, personnel procedures, etc.

2. Train and educate all personnel on the contamination sensitivity of the hardware they are handling or are in proximity to.

3. Perform detailed contamination modeling analyses. The results of these detailed models are useful in determining the most critical surfaces and what activities and/or materials are the biggest threats to maintaining their optical performance.

4. Develop detailed on-orbit contamination control procedures to limit exposure from deleterious environments. For the HST this was the servicing mission. For the Scientific Instruments this was subsequent servicing missions. For the STIS it also included bright object avoidance.

5. Develop non-metallic materials guidelines early in the program. For contamination-sensitive hardware, use outgassing data (ASTM E1559 or equivalent) in conjunction with contamination modeling to assess deposition thicknesses and, subsequently, long-term optical performance.

6. Develop cleaning procedures for metallic hardware and processes early in the program which reduces the amount of molecular contamination to the monolayer level. No additional cleanliness is gained from preprocessing this hardware under vacuum conditions. This is both a cost and schedule reduction for contamination-sensitive hardware.

\section{Multi-Mission Hardware}

1. Store, integrate, and test multi-mission hardware in stringently controlled environments, preferably a cleanroom. When not in a cleanroom, double bag hardware with approved bagging material.

2. Control the type and amount of all added materials to the multi-mission hardware so that outgassing limits are not violated. Verify, by test, that the batch of material used will not be a significant contamination source.

3. Certify outgassing levels of added (new) hardware at the sub-assembly levels prior to integration onto the multi-mission hardware.

4. Maintain surface cleanliness levels during storage or low work periods. Periodic cleaning is required for multi-mission hardware that is not bagged. 


\section{Scientific Instruments}

1. Design the instrument to provide some isolation of the contamination-sensitive optical elements from known contaminant sources (e.g., electronics boxes). This reduces the number of subassembly bakeouts and system-level outgassing periods.

2. Develop an overall contamination control program. Flow down the requirements into contamination control plans, handling procedures, personnel procedures, etc.

3. Select non-metallic materials based on low-outgassing requirements.

4. Limit the amount of non-metallic materials near contamination-sensitive surfaces.

5. Develop cleaning procedures for metallic hardware and processes early in the program which reduces the amount of molecular contamination to the monolayer level. No additional cleanliness is gained from preprocessing this hardware under vacuum conditions. This is both a cost and schedule reduction for contamination-sensitive hardware.

6. Pre-process large amounts of low-outgassing materials (such as Braycote 601) prior to use to further reduce on-orbit outgassing when used near contamination-sensitive surfaces. This was successful in limiting the contribution from approximately 5-1bm (approximately 2-kg) of Braycote 601 used on the STIS optical mechanisms (9).

7. Train and educate all personnel on the contamination sensitivity of the hardware they are handling or are in proximity to.

8. Monitor contamination-sensitive optics using optical witness samples. These monitoring programs are useful in detecting contamination events (or lack thereof).

9. Develop bakeout programs to maintain the UV capability of contamination-sensitive instruments

10. Perform detailed contamination modeling analyses. The results of these detailed analyses are useful in determining the most critical surfaces and what activities and/or materials are the biggest threats to maintaining their optical performance.

11. Develop detailed contamination controls for on-orbit servicing activities. For the HST scientific instruments, this included the mission in which they were installed and all subsequent servicing missions.

12. Develop detailed contamination procedures for on-orbit operations. These plans should include bright object avoidance, spacecraft checkout, etc.

\section{SUMMARY}

The HST Contamination Control Program has been demonstrated to be a successful approach to maintaining long-term science capability while controlling costs through the reuse of multi-mission hardware and mission-unique trade studies and contamination controls. By maintaining the cleanliness of the hardware between missions, and by controlling the materials added to the hardware during modification and refurbishment, both project funding for contamination recertification and schedule were significantly reduced. The lessons learned, while unique to the HST and the Orbiter, are applicable in many instances to other contamination-sensitive spacecraft and other launch vehicles.

The HST Contamination Control Program utilized detailed contamination modeling and trade studies to compare the risk of potentially contaminating the HST and its scientific instruments during servicing mission activities. The results were used to craft the Flight Rules for each servicing mission. Modeling was also used to perform trade studies for the scientific instruments, starting at the design stage through the test and integration phase to compare such things as the preferred venting path which produced the least deposition to the contaminationsensitive optics.

Feedback, through the periodic on-orbit calibration of the scientific instruments and post-mission inspections and testing for the returned scientific instruments, allowed the HST Contamination Control Program to incorporate new contamination controls as necessary to preserve the HST science capability. Data from the scientific instruments, such as STIS, provided indirect measurements of the HST contamination levels. This in turn, was used to determine the best contamination control approach for new hardware (scientific instruments and orbital replacement units). 


\section{REFERENCES}

1. Hansen, P. A., Day, D. T., Secunda, M. S., and Rosecrans, G. P., "The Swift Project Contamination Control Program: A Case Study of Balancing Cost, Schedule and Risk", Proceedings of the $21^{\text {st }}$ Aerospace Tesing Seminar, October 2003.

2. Hedgeland, R. J., Hansen, P. A., and Hughes, D. W., "An Integrated Approach for Contamination Control and Verification for the Hubble Space Telescope First Servicing Mission", SPIE 2216: 10-21, July 1994.

3. Hansen, P. A., et. al., "Hubble Space Telescope Second Servicing Mission Contamination Control Program", SPIE 2864: 27-35, August 1996.

4. Hansen, P. A., The Hubble Space Telescope Servicing Mission 3A Contamination Control Program", Proceedings of the ${ }^{\text {th }}$ International Symposium on 'Materials in Space Environment', June 2000.

5. Hedgeland, R. and Hansen, P, "Contamination Design of a Scientific Instrument Protective Enclosure for the Hubble Space Telescope Servicing Mission", AIAA paper no. 93-1102, 1993.

6. Hansen, P., Hughes, D., Hedgeland, R., Studer, R., Kostos, K., and Chivatero, C., "Hubble Space Telescope Servicing Mission Scientific Instrument Protective Enclosure Design Requirements and Contamination Controls', Proceeding of the IES $18^{\text {th }}$ Space Simulation Conference, October 1994.

7. Hughes, D., Hedgeland, R., Geer, W., and Greenberg, B, "Maintaining a Class M 5.5 Environment in a Class M 6.5 Cleanroom", SPIE 2261: 46-57, July 1994.

8. Hansen, P., Hughes, D., Montt, K., and Triolo, J, "Innovative Contamination Certification of Multi-Mission Flight Hardware", $20^{\text {th }}$ Space Simulation Conference The Changing Paradigm Proceedings, NASA/CR1998-208598 Preprint: 1-13, October 1998.

9. Hansen, P. A., "Correlation of the Hubble Space Telescope (HST) Space Telescope Imaging Spectrograph (STIS) On-orbit Data with Pre-launch Predictions and Ground Contamination Controls", Proceedings of the ESA Contamination of Optical Equipment Workshop, December 2003.

10. Hansen, P., Hedgeland, R., Maag, C., and Seaman, C., "Results of STS-51 Orbiter Crew Contamination Generation and EVA Payload Bay Transfer Experiment", SPIE 2261: 2-9, July 1994.

11. Tveekrem, J., Leviton, D., Fleetwood, C., and Feinberg, L., "Contamination-induced Degradation of Optics Exposed to the Hubble Space Telescope Interior", SPIE 2864: 246-257, August 1996.

12. Tveekrem, J., Contamination Effects on EUV Optics, NASA/TP-1999-209264, June 1999.

13. Profitt, C. R., Brown, T. M., Mobasher, B., and Davies J., "Absolute Flux Calibration of STIS MAMA Imaging Modes", Instrument Science Report STIS 2003-01, January 28, 2003.

14. Product Cleanliness Levels and Contamination Control Program, IEST-STD-CC1246D, Institute of Environmental Sciences and Technology, December 2002.

15. Standard Test Method for Contamination Outgassing Characteristics of Spacecraft Materials, ASTM E1559-00, American Society for Testing and Materials, 2000. 